\title{
Generation of orthotopic patient-derived xenografts from gastrointestinal stromal tumor
}

\author{
Jason K Sicklick*, Stephanie Y Leonard ${ }^{1}$, Michele L Babicky ${ }^{1}$, Chih-Min Tang ${ }^{1}$, Evangeline S Mose ${ }^{1}$, \\ Randall P French', Dawn V Jaquish', Carl K Hoh ${ }^{2}$, Michael Peterson ${ }^{3}$, Richard Schwab ${ }^{4}$ and Andrew M Lowy ${ }^{1}$
}

\begin{abstract}
Background: Gastrointestinal stromal tumor (GIST) is the most common sarcoma and its treatment with imatinib has served as the paradigm for developing targeted anti-cancer therapies. Despite this success, imatinib-resistance has emerged as a major problem and therefore, the clinical efficacy of other drugs has been investigated. Unfortunately, most clinical trials have failed to identify efficacious drugs despite promising in vitro data and pathological responses in subcutaneous xenografts. We hypothesized that it was feasible to develop orthotopic patient-derived xenografts (PDXs) from resected GIST that could recapitulate the genetic heterogeneity and biology of the human disease.

Methods: Fresh tumor tissue from three patients with pathologically confirmed GISTs was obtained immediately following tumor resection. Tumor fragments $\left(4.2-\mathrm{mm}^{3}\right)$ were surgically xenografted into the liver, gastric wall, renal capsule, and pancreas of immunodeficient mice. Tumor growth was serially assessed with ultrasonography (US) every 3-4 weeks. Tumors were also evaluated with positron emission tomography (PET). Animals were sacrificed when they became moribund or their tumors reached a threshold size of 2500- $\mathrm{mm}^{3}$. Tumors were subsequently passaged, as well as immunohistochemically and histologically analyzed.
\end{abstract}

Results: Herein, we describe the first model for generating orthotopic GIST PDXs. We have successfully xenografted three unique KIT-mutated tumors into a total of 25 mice with an overall success rate of $84 \%(21 / 25)$. We serially followed tumor growth with US to describe the natural history of PDX growth. Successful PDXs resulted in 12 primary xenografts in NOD-scid gamma or NOD-scid mice while subsequent successful passages resulted in 9 tumors. At a median of 7.9 weeks (range 2.9-33.1 weeks), tumor size averaged 473 $\pm 695-\mathrm{mm}^{3}$ (median 199- $\mathrm{mm}^{3}$, range 12.6-2682.5- $\mathrm{mm}^{3}$ ) by US. Furthermore, tumor size on US within 14 days of death correlated with gross tumor size on necropsy. We also demonstrated that these tumors are FDG-avid on PET imaging, while immunohistochemically and histologically the PDXs resembled the primary tumors.

Conclusions: We report the first orthotopic model of human GIST using patient-derived tumor tissue. This novel, reproducible in vivo model of human GIST may enhance the study of GIST biology, biomarkers, personalized cancer treatments, and provide a preclinical platform to evaluate new therapeutic agents for GIST.

Keywords: GIST, Imatinib, KIT, NOD-scid, NOD-scid gamma, PDX, Sarcoma, Targeted therapy

\footnotetext{
* Correspondence: jsicklick@ucsd.edu

'Division of Surgical Oncology and Department of Surgery, Moores UCSD Cancer Center, University of California, San Diego, 3855 Health Sciences Drive, Mail Code 0987, La Jolla, CA 92093-0987, USA

Full list of author information is available at the end of the article
} 


\section{Background}

Gastrointestinal stromal tumor (GIST), the most common gastrointestinal mesenchymal tumor, afflicts 12-20 patients per million annually [1]. Unlike many other cancers, the genomic and molecular events driving GIST are well characterized. These include mutations in several protein kinase genes including KIT, PDGFR $\alpha$, and BRAF that are known to regulate fundamental processes in oncogenesis including tumor proliferation, metastasis, neo-vascularization, and chemo-resistance [2-4]. GIST has served as a paradigm for the development of targeted cancer therapies because inhibition of KIT and PDGFR $\alpha$ has resulted in therapeutic benefit. At present, the first-line treatment for patients with metastatic, unresectable or resected high-risk GIST is imatinib (Gleevec, Novartis, Basel, Switzerland) [5-7], a small molecule inhibitor of tyrosine kinases including KIT (c-KIT, CD117) and BCR-ABL. This drug has been shown to have profound therapeutic benefit with a favorable toxicity profile. Because of these qualities, imatinib is often cited as the prototype for targeted therapeutics development.

Beyond our knowledge that KIT mutations drive GIST sarcomagenesis, it is now known that specific KIT mutations are both prognostic and predictive of responses to the current kinase inhibitors. For example, KIT Exon 9 mutations are associated with more aggressive phenotypes and imatinib insensitivity as compared to KIT exon $11 \mathrm{mu}-$ tations $[4,8]$. Secondary resistance to imatinib, which occurs in half of patients after 20 months of therapy, is most commonly caused by acquired, non-randomly distributed single nucleotide KIT mutations within the ATP binding pocket (exons 13 and 14) and the kinase activation loop (exons 17 and 18) [9-12]. Sunitinib (Sutent, Pfizer, New York, NY), a multikinase inhibitor with activity against PDGFR, VEGFR and KIT, is employed as second line therapy for GIST. Clinical trials have shown that in imatinibresistant cases, only $12-19 \%$ of sunitinib-treated patients have significant responses [13,14]. These anti-GIST therapies were developed based upon efficacy data in vitro or in vivo using subcutaneous models of tumor implantation. However, once a patient progresses on sunitinib, treatment options are limited as evidenced by two recent, large clinical trials which reported on the efficacy of dasatinib (Bristol-Myers Squibb, New York, NY), a combined Src and BCR-ABL inhibitor, and regorafenib (Bayer, Pittsburgh, PA), a combined VEGFR2 and TIE2 inhibitor $[15,16]$. Dasitinib failed to show any benefit in this patient population while in the Phase III GRID trial of regorafenib, $62 \%$ of patients developed resistance to the drug, and consequently disease progression by the sixth month of therapy [17]. This highlights the urgency for developing more effective agents to treat GIST [18], as well as more broadly applicable preclinical models to accomplish this goal.
Despite the importance of preclinical studies on GIST tumorigenesis and resistance mechanisms, there are currently limited model systems for studying this disease in vitro and in vivo. For instance, two GIST cell lines with KIT exons 11 and 13 mutations have been reported in the literature; $[19,20]$ however, the second most common KIT mutation (e.g., exon 9) lacks a corresponding cell line for in vitro assays. Moreover, there are no cell lines which contain any exon 14 or 18 mutations while most of the common exon 17 mutations are not present in cell lines except with overexpression vectors often used in non-GIST lines, such as BaF3 cells. In addition, no cell lines exist which contain either PDGFR $\alpha$ mutation/ deletions/insertions or $\mathrm{BRAF}^{\mathrm{V} 600 \mathrm{E}}$ mutations that also cause GIST. Regarding mouse models of GIST, subcutaneous (SQ) xenografts have been utilized as the prototype in nude mice [21-23]. However, because tumor growth or responses to drug treatment observed in SQ xenograft models are often different from those observed in an orthotopic environment, two groups have developed transgenic mouse models of GIST. Rubin and colleagues identified a $\mathrm{KIT}^{\mathrm{K} 641 \mathrm{E}}$ mutation (exon 13) in sporadic human GISTs and in the germ line of familial GIST syndrome patients [24]. They then generated homozygous and heterozygous $K I T^{\mathrm{K} 641 \mathrm{E}}$ transgenic mice that develop cecal GISTs with complete penetrance. However, in humans, cecal (e.g., colonic) GISTs are quite rare, suggesting that this model does not completely recapitulate the human disease. Additionally, Besmer and colleagues developed a second model via a knock-in strategy by introducing a KIT exon 11 mutation ( $5558^{\Delta /+}$ ) into the mouse genome [25]. While the latter transgenic model is more representative of the human disease, it only embodies a mutation that is well studied, evaluable in the GIST-T1 cell line, and sensitive to imatinib.

Despite the aforementioned models, there remains a gap in our ability to predict effective drugs or study the biology of the less frequent, but often drug-resistant, gene mutations in GIST. Therefore, our goal was to develop a reproducible, orthotopic patient-derived xenograft model of GIST. This novel model for studying GIST in vivo recapitulates the intra-abdominal microenvironment in which GIST arises and allows for the study of the increasingly appreciated heterogeneity in the biology of GIST. It is our hope that this model may serve as a valuable resource for personalized cancer therapy and the evaluation of new therapeutic agents for GIST.

\section{Materials and methods}

\section{Animal studies}

NOD-scid (NS) and NOD-scid IL2Rgamma ${ }^{\text {null }}$ (NOD-scid gamma, NSG) mice at 8-10 weeks of age were obtained from the Jackson Laboratory (Bar Harbor, Maine). NS homozygous mice harboring a spontaneous Prkdc ${ }^{\text {scid }}$ 
mutation (commonly referred to as scid) are a model for severe combined immune deficiency characterized by an absence of functional $\mathrm{T}$ cells and $\mathrm{B}$ cells, hypogammaglobulinemia, lymphopenia, and a normal hematopoietic microenvironment. NSG mice combine the features of the NOD/ShiLtJ background, the severe combined immune deficiency mutation and an IL2 receptor gamma chain deficiency. As a result, this NSG strain (NOD.Cg-Prkdc ${ }^{\text {scid }}$ Il2 $\left.\mathrm{rg}^{\mathrm{tm} 1 \mathrm{~W} / \mathrm{l}} / \mathrm{SzJ}\right)$, lacks functional $\mathrm{T}$ cells, B cells, and NK cells, as well as is deficient in cytokine signaling. Consequently, this NSG strain performs better in engraftment of human hematopoietic stem cells and peripheral-blood mononuclear cells than any other published mouse strains [26-31]. Moreover, these recent publications have demonstrated this strain's utility in the study of solid tumor xenografts and cancer stem cell engraftment.

All research mice were maintained in a barrier facility under HEPA-filtered air with food and water available ad libitum. Food, water and cage bedding were sterilized prior to use. Temperature $\left(20-21^{\circ} \mathrm{C}\right)$, humidity $(50-60 \%)$ and 12-hour light-dark cycle were controlled. Animals were manipulated under sterile conditions during surgery. Animal experiments fulfilled National Institutes of Health and University of California, San Diego (UCSD) requirements for humane animal care. The UCSD Institutional Animal Care and Use Committee approved experimental methods.

\section{Sourcing of human tumor tissue}

Tumor acquisition/banking is routinely performed for all GIST operations under our Institutional Review Board (IRB) approved protocol (\#090401). Written informed consent was obtained from all patients prior to sample collection. Three patients with KIT-mutated GIST underwent operations in 2011. All patients' demographics were listed in Table 1. The tumor tissue for xenografts was obtained at the time of tumor resection after a pathologist acquired tissue that was needed for the patient's routine clinical care and confirmed the histologic diagnosis. Additional tissue was banked in our biorepository. Excess fresh tumor was used for immediate xenografting into mice. All surgically resected tumor fragments were stored in sterile specimen cups and expeditiously transported from the operating room to our laboratory on ice. Staining for clinical diagnosis included hematoxylin and eosin (H\&E), KIT and DOG1. Genetic materials derived from tumors were analyzed by ARUP Laboratories (Salt Lake City, UT) for KIT and PDGFR $\alpha$ mutations.

\section{Implantation of patient-derived xenografts}

Tumor was dissected into $2 \times 2 \mathrm{~mm}$ fragments $\left(4.2 \mathrm{~mm}^{3}\right)$ and placed in a petri dish kept on ice containing sterile, antibiotic-free DMEM media (Mediatech, Manassas, VA)
Table 1 Patient demographics

\begin{tabular}{|c|c|}
\hline & Number \\
\hline \multicolumn{2}{|l|}{ Age } \\
\hline Mean & 62 years \\
\hline Range & $46-72$ years \\
\hline \multicolumn{2}{|l|}{ Gender } \\
\hline Male & 2 \\
\hline Female & 1 \\
\hline \multicolumn{2}{|l|}{ Location of primary tumor } \\
\hline Stomach & 0 \\
\hline Small bowel & 3 \\
\hline Duodenum & 1 \\
\hline Jejunum & 1 \\
\hline Ileum & 1 \\
\hline \multicolumn{2}{|l|}{ Presentation } \\
\hline Abdominal pain & 2 \\
\hline Bleeding & 1 \\
\hline Recurrence/metastasis on CT scan & 1 \\
\hline \multicolumn{2}{|l|}{ Previous imatinib therapy } \\
\hline Yes & 1 \\
\hline No & 2 \\
\hline \multicolumn{2}{|l|}{ Primary tumor size } \\
\hline Mean & $19.2 \mathrm{~cm}$ \\
\hline Range & $6.5-38.0 \mathrm{~cm}$ \\
\hline \multicolumn{2}{|l|}{ Mitotic index (mitoses per $5 \mathrm{~mm}^{2}$ ) } \\
\hline Mean & 32.7 \\
\hline Range & $10-65$ \\
\hline \multicolumn{2}{|l|}{ Peritoneal metastases } \\
\hline Yes & 2 \\
\hline No & 1 \\
\hline \multicolumn{2}{|l|}{ KIT exon mutational analysis } \\
\hline Exon 9 & 2 \\
\hline Exon 11 & 1 \\
\hline Exon $13 / 14$ & 0 \\
\hline Exon $17 / 18$ & 0 \\
\hline
\end{tabular}

until implantation. NS and NSG mice were anesthetized with intraperitoneal injection of ketamine:xylazine cocktail $(60-\mathrm{mg} / \mathrm{kg}: 10-\mathrm{mg} / \mathrm{kg})$. They were then placed in the supine position on a warm pad to maintain body temperature. Once mice were sedated, the abdominal wall was shaved and cleansed with $70 \%$ alcohol and betadine. A 1-2-cm midline incision was made through the skin, fascia and peritoneum. Surgical sutures (6-0 silk) were used to implant $2 \times 2 \mathrm{~mm}$ tumor fragments onto the livers, gastric walls, renal capsules, or lesser sacs (peri-pancreatic area). Organs implanted with tumor fragments were returned to the abdomen and the 
peritoneum and the skin were closed with 6-0 Prolene suture. A total of 14 animals underwent initial tumor implantation of freshly dissected human tumor tissues.

Mice were monitored daily for 5 consecutive days after surgery with particular attention paid to animal distress, wound dehiscence, and signs of infection. Thereafter, they were examined 2-3 times per week. Three researchers (SL, CT and EM) assessed tumor progression by palpation twice a week. Tumor progression was also evaluated by ultrasound every 3-4 weeks as described in the "Tumor Imaging" section. Animals were euthanized based on either tumor volume (threshold $2500 \mathrm{~mm}^{3}$ ) as determined by ultrasound or clinical status during the observation period as specified in our IACUC-approved protocol. A necropsy was performed on the animals after euthanasia to assess the presence and distribution of tumors. Tumors were harvested and fixed in 10\% formalin for histological and immunohistological analyses. Harvested tumors were also subject to serial passages into additional 11 mice. All 3 patient-derived xenografts were successfully passaged up to twice in order to determine the ability to perpetuate and expand these tumors for extended periods of time. This provides the potential for developing a model that is based upon a small amount of available tumor, which can be utilized for current and future studies. Mouse characteristics for tumor implantation are listed in Table 2.

\section{Tumor imaging}

Tumors were serially imaged with Visual Sonics Vevo 770 ultrasound machine (VisualSonics Inc, Toronto, Canada) every 3-4 weeks by a single, experienced ultrasonographer. Mice were kept anesthetized using continuous isoflurane inhalation. Prior to ultrasonography, abdominal wall hair was removed from the skin overlying the tumor implant area with clippers and hair removal cream. Their skin was then covered with an aqueous ultrasonic gel and a high frequency transducer (RMV706) at 20-60 MHz range was used for imaging. Tumor detection was recorded as cine loops of ultrasound images. Digital images were reviewed to select the tumors' largest cross-sectional diameters (e.g., length and width) from a single image frame. Tumor volume was calculated as $(\pi / 6) \times(\text { length } \times \text { width })^{3 / 2}$ [32]

Two animals were also evaluated with Positron Emission Tomography (PET, GE eXplore Vista DR PET scanner). Mice were anesthetized by isoflurane inhalation and placed on an imaging bed controlled by a computer in order to insert into the ring-type gantry. They were then administered a radiopharmaceutical fluorodeoxyglucose[18F] (F-18 FDG) delivered by tail vein injection. F-18 FDG is used for the assessment of glucose metabolism, and therefore serves as an indicator for high metabolic activity of tissue, such as malignant tumors. One mouse was given $250 \mu \mathrm{Ci}$ in $113 \mu \mathrm{L}$ while another mouse
Table 2 Mouse characteristics for generation of GIST patient-derived xenografts

\begin{tabular}{|c|c|c|}
\hline & Number & Percent (\%) \\
\hline \multicolumn{3}{|l|}{ Age at implantation } \\
\hline Mean \pm standard deviation & $9.1 \pm 7.3$ weeks & \\
\hline Range & $5.7-30.6$ weeks & \\
\hline \multicolumn{3}{|l|}{ Gender } \\
\hline Male & 8 & $32.0 \%$ \\
\hline Female & 17 & $68.0 \%$ \\
\hline \multicolumn{3}{|l|}{ Genotype } \\
\hline NOD-scid (NS) & 10 & $40.0 \%$ \\
\hline NOD-scid gamma (NSG) & 15 & $60.0 \%$ \\
\hline \multicolumn{3}{|l|}{ Tumor development } \\
\hline Yes & 21 & $84.0 \%$ \\
\hline No & 4 & $16.0 \%$ \\
\hline \multicolumn{3}{|l|}{ Passages } \\
\hline 0 (Primary, successes/total) & $11 / 14$ & $85.7 \%$ \\
\hline 1 (Successes/total) & $5 / 6$ & $83.3 \%$ \\
\hline 2 (Successes/Total) & $5 / 5$ & $100.0 \%$ \\
\hline \multicolumn{3}{|l|}{ Location of implantation } \\
\hline Successful implantations/total & $21 / 25$ & $84.0 \%$ \\
\hline Liver (successes/total) & $9 / 10$ & $90.0 \%$ \\
\hline Renal capsule (successes/total) & $9 / 10$ & $90.0 \%$ \\
\hline Lesser sac (successes/total) & $2 / 3$ & $66.7 \%$ \\
\hline Gastric wall (successes/total) & $1 / 2$ & $50.0 \%$ \\
\hline
\end{tabular}

received $395 \mu \mathrm{Ci}$ in $200 \mu \mathrm{L}$. A sequence of successive whole-body images was acquired in the 3D mode using the system software. DICOM images were analyzed by an experienced nuclear medicine radiologist $(\mathrm{CKH})$ for tumor standardized uptake values (SUVs).

\section{Histopathology and immunohistochemistry}

Serial sections of formalin-fixed, paraffin-embedded (FFPE) resected xenografts, $10-\mu \mathrm{m}$ thick, were used for histopathology analyses by H\&E staining. For all tumors, the histological diagnoses were confirmed under light microscopy by an experienced pathologist. Sections for immunohistochemical staining were treated twice with $0.3 \%$ Triton X-100 (Sigma-Aldrich) in PBS for 10 minutes and then in $0.3 \%$ hydrogen peroxide solution in order to block endogenous peroxidase activity. The sections were then blocked with serum followed by an Avidin-Biotin blocking reagent (Vector Laboratories; Burlingame, CA) in order to inhibit non-specific binding in the tissue. The sections were then incubated with polyclonal rabbit anti-human CD117 (c-KIT) antibody (1:50, Dako North America, Carpinteria, CA) overnight at $4^{\circ} \mathrm{C}$. Sections were next incubated with biotinylated secondary antibody and $\mathrm{ABC}$ reagents of the Vectastain 
Elite Universal $\mathrm{ABC}$ kit according to the manufacturer's instructions (Vector Laboratories). The secondary antibody was detected using the Avidin-Biotin-Peroxidase method with 3,3'-diaminobenzidine as the substrate (Vector Laboratories). Negative controls were performed by omitting the primary antibody and/or using isotype control antibody.

\section{Statistical analysis}

Comparisons between groups were performed using Prism 4. (GraphPad, La Jolla, CA). Results are reported as mean \pm standard deviation (s.d.) or standard error of the mean (s.e.m.) as appropriate. Comparison data were analyzed for significance using the Student's t-test, ANOVA, and Bonferroni's multiple comparison test. Statistical significance was accepted at the 5\% level and statistical trends were accepted at the $10 \%$ level.

\section{Results}

\section{Sourcing human GISTs}

To our knowledge, only subcutaneous (SQ) GIST xenografts have been performed in mice. We hypothesized that human GISTs could be intraperitoneally (IP) xenografted into immunodeficient mice in order to better recapitulate the biology of GIST, a disease which tends to metastasize to the liver and peritoneum, but not the soft tissue of the flank. KIT-mutated GIST tissue from three patients was used for xenografts in this study
(Table 1). This included tumors from 2 male patients and 1 female patient with mean age of 62 . Their primary tumors were all found in the small bowel. One patient had a clinical presentation of worsening abdominal pain while the second patient presented with acute onset abdominal pain due to intratumoral bleeding. The third patient had GIST recurrence and metastatic tumors detected by CT scan. Only the latter patient had previously received imatinib therapy. The mean tumor size was $19.2 \mathrm{~cm}$ (range: $6.5-38.0 \mathrm{~cm}$ ) with an average mitotic index (mitoses per 50 high-power fields equals mitoses per $5 \mathrm{~mm}^{2}$ ) of 32.7 (range: 10 - 65). Based upon pathological examination, one patient had stage IIIB and the other two patients had stage IV GIST with peritoneal involvement. Genetic sequencing analyses revealed that two tumors had KIT exon 9 mutations and one tumor had an exon 11 mutation. Herein, we present a representative case of a 46-year old male patient (Figure 1). The patient was first examined by CT scan and found to have a heterogeneous tumor mass in the left upper quadrant of the abdomen (Figure 1A) which was FDG-avid on PET-CT scan (Figure 1B). He underwent surgical resection of a $13.0 \times 11.0 \times 10.0 \mathrm{~cm}$ GIST removed from the fourth portion of the duodenum and the proximal jejunum (Figure 1C). Histologically the tumor tissue had strong KIT (Figure 1D) and DOG-1 staining (data not shown), consistent with GIST. This tumor had mixed spindle cell and epithelioid histology, as well as a mitotic index of 23
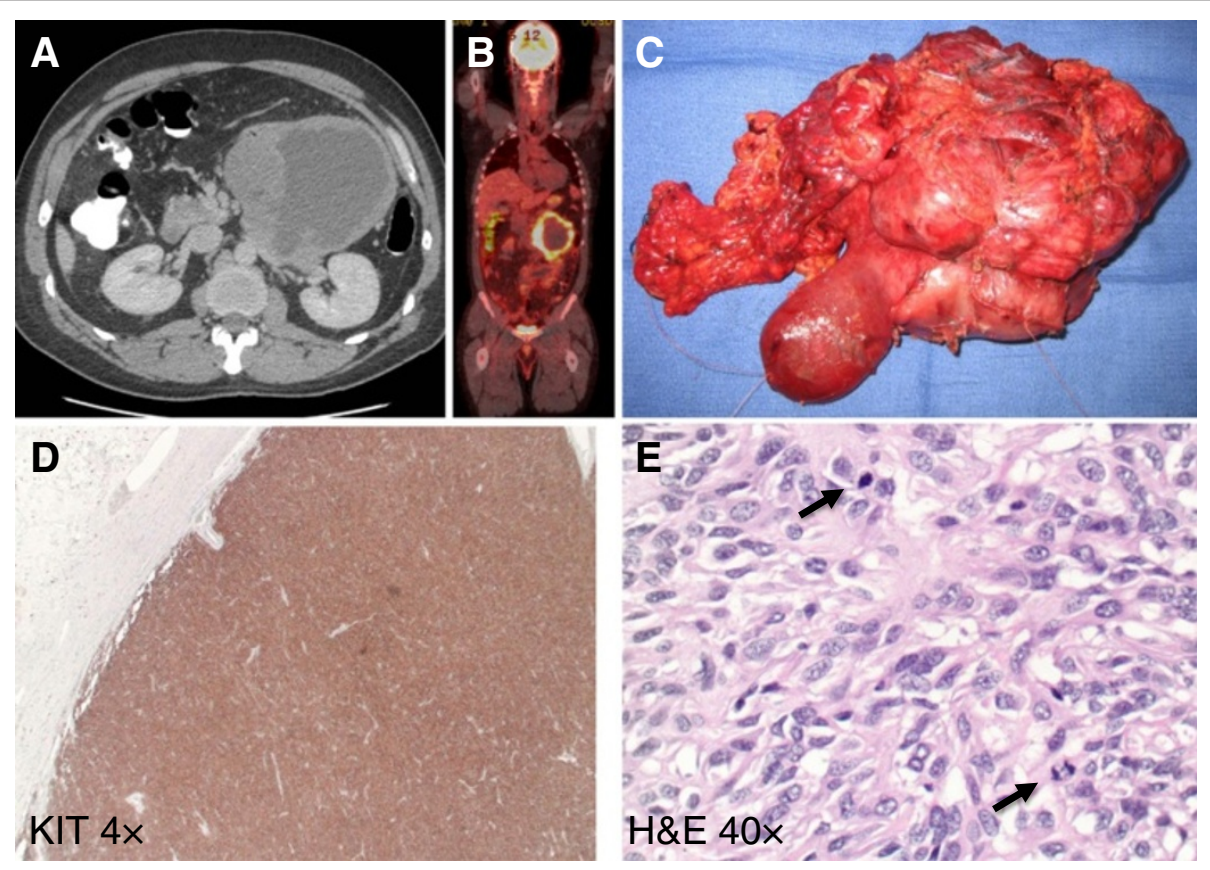

Figure 1 Resection of a 13-cm GIST from a 46-year old man that presented with abdominal pain. A. CT of the abdomen demonstrates a heterogeneous mass in the left abdomen. B. PET-CT demonstrating an FDG-avid mass in the left upper quadrant. C. Gross specimen following resection of the third and fourth portions of the duodenum and proximal jejunum where the tumor was arising. $\mathbf{D}$. Pathology consistent with a GIST with strong KIT immunostaining. E. H\&E staining demonstrating 23 mitoses (arrows) per 50 high power fields. 
per 50 high-power fields (hpf) (Figure 1E). Similarly, the other two tumors also had high-risk features.

\section{Development of GIST PDXs}

To develop a novel xenograft model of GIST in vivo, fresh human tumor tissues were implanted within immunodeficient mice. We employed a midline laparotomy to suture $2 \times 2 \mathrm{~mm}$ tumor fragments into the abdominal viscera of NS $(\mathrm{N}=10)$ and NSG $(\mathrm{N}=15)$ mice. This included 14 primary xenografts and 11 passaged xenografts. Fresh tumor tissues implanted into 14 mice were defined as Passage zero (P0). Tumor tissues were harvested from P0 mice and implanted into 6 mice as Passage 1 (P1); and subsequently another xenograft with P1 tumors was carried out in 5 mice as Passage 2 (P2). Xenografts were performed in 25 mice with an $84 \%$ $(21 / 25)$ success rate which included a $4 \%(1 / 25)$ perioperative mortality in a P2/NS mouse. Different implantation sites were compared for xenograft efficiency. We observed tumor growth and progression in the liver $(9 / 10)$, renal capsule $(9 / 10)$, lesser sac $(2 / 3)$, and gastric wall $(1 / 2)$. There was no tumor growth in three mice with the following characteristics: P0/NSG/Kidney; P1/ NSG/Liver; and P0/NS/Stomach. Detailed characteristics of the mice used for the PDXs are shown in Table 2.

\section{Natural history of GIST orthotopic PDXs}

Given the intra-abdominal location of tumors, standard calipers cannot be employed to monitor tumor growth. Therefore, in order to monitor the natural history of tumor progression, ultrasound (US) imaging was conducted every 3-4 weeks after implantation. As shown in Figure $2 \mathrm{~A}$, one tumor in the liver reached $7 \times 2.4 \mathrm{~mm}$ in size as determined by US at 4 weeks. By 7 weeks, the same mouse had to be terminated due to poor health. The tumor was harvested (Figure 2B-C) and passaged into additional NS mice. In the entire cohort, PDX tumor size (as determined by US imaging) at 2.9-33.1 weeks (median 7.9 weeks) averaged $473 \pm 695 \mathrm{~mm}^{3}$ (median $199 \mathrm{~mm}^{3}$, range 12.6-2682.5 $\mathrm{mm}^{3}$ ). Every surviving mouse with a PDX received at least 2 serial US studies (mean $2.5 \pm 1.7$, median 2, range $0-7$ ) in order to monitor the natural history of their tumor growth. Based upon maximum tumor size achieved, we could sort tumors into two groups with distinct tumor growth patterns (Figure 3). In general, tumors with a maximum tumor size $\geq 50 \mathrm{~mm}^{3}$ (Figure $3 \mathrm{~A}, \mathrm{~N}=11$ ) tended to be faster growing than those with a maximum tumor size $<50 \mathrm{~mm}^{3}$ (Figure $3 \mathrm{~B}, \mathrm{~N}=10$ ). However, in the former group, there were outliers that began growing slowly but later achieved a larger final tumor volume.

To further assess the how tumor passage, immunodeficient mouse type, and tumor implantation location affected maximum tumor size, we performed subgroup analyses of the 21 mice that developed tumors (Figure 4). P1-2 tumors $\left(829.5 \pm 326.1 \mathrm{~mm}^{3}, \mathrm{~N}=9\right)$ were larger than P0 tumors $\left(187.1 \pm 59.4 \mathrm{~mm}^{3}, \mathrm{~N}=12, \mathrm{P}<0.05\right.$, Figure 4A). NS tumors $\left(994.1 \pm 329.7 \mathrm{~mm}^{3}, \mathrm{~N}=8\right)$ were larger than NSG tumors $\left(135.2 \pm 56.7 \mathrm{~mm}^{3}, \mathrm{~N}=13\right.$, $\mathrm{P}<0.006$, Figure 4B). Furthermore, $\mathrm{P} 1-2 / \mathrm{NS}$ tumors $\left(1240.0 \pm 392.5 \mathrm{~mm}^{3}, \mathrm{~N}=6\right)$ were larger than $\mathrm{P} 0 / \mathrm{NSG}$ tumors $\left(173.2 \pm 69.9 \mathrm{~mm}^{3}, \mathrm{~N}=10, \mathrm{P}<0.01\right)$ and $\mathrm{P} 1-2$ / NSG tumors $\left(8.6 \pm 3.6 \mathrm{~mm}^{3}, \mathrm{~N}=3, \mathrm{P}<0.05\right.$, Figure $\left.4 \mathrm{C}\right)$. There was no difference between $\mathrm{P} 1-2 / \mathrm{NS}$ tumors and P0/NS tumors $\left(256.4 \pm 87.3 \mathrm{~mm}^{3}, \mathrm{~N}=2\right)$ due to the small sample size of the latter group. Comparison of the maximum tumors sizes of the four implantation locations (Kidney: $610.9 \pm 338.5 \mathrm{~mm}^{3}, \mathrm{~N}=9$; Liver $284.7 \pm 109.1$ $\mathrm{mm}^{3}, \mathrm{~N}=9$; Pancreas $601.6 \pm 554.5 \mathrm{~mm}^{3}, \mathrm{~N}=2$; Stomach $446.4 \mathrm{~mm}^{3}, \mathrm{~N}=1$ ) showed no statistically significant differences due to variability within the groups, confounding factors such as passage/mouse type, or small sample sizes (Figure 4D). However, in subgroup analyses, the P1-2/NS mice kidney tumors $\left(2347.6 \pm 334.9 \mathrm{~mm}^{3}\right.$, $\mathrm{N}=2$ ) tended to be larger than liver tumors (770.6 \pm $\left.191.5 \mathrm{~mm}^{3}, \mathrm{~N}=2, \mathrm{P}<0.055\right)$ and pancreas tumors $601.6 \pm$ $554.5 \mathrm{~mm}^{3}, \mathrm{~N}=2, \mathrm{P}<0.12$, Figure $\left.4 \mathrm{E}\right)$. To determine the accuracy of our ultrasound findings, we compared the
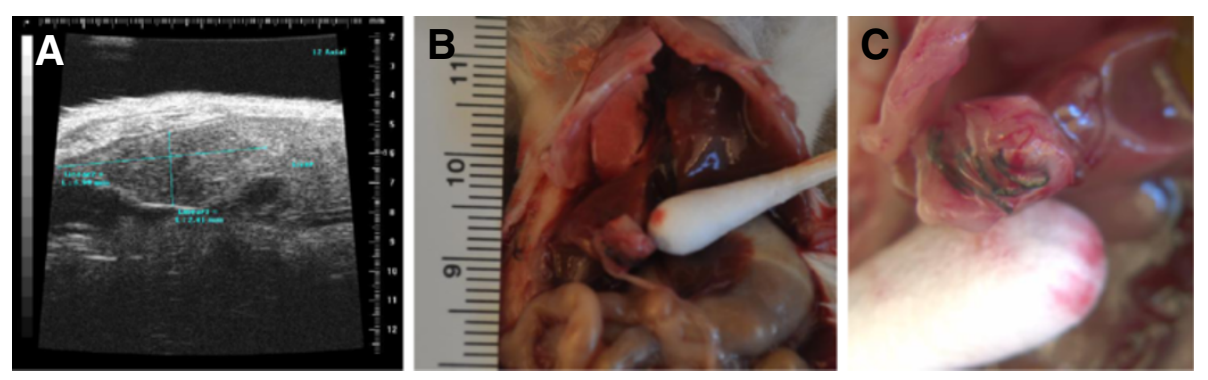

Figure 2 Generation of GIST xenografts. Tumor was xenografted into the livers, kidneys, lesser sacs and gastric walls of NOD-scid and NSG mice. Using ultrasound, we followed tumor progression. A. At 4 weeks, one tumor in the liver increased from $4 \times 2 \mathrm{~mm}$ to $7 \times 2.4 \mathrm{~mm}$ based upon ultrasound examination. B-C. At 7 weeks, the same mouse became distressed and we performed a necropsy with passage of the tumor into two NOD-scid mice. The tumor is noted at the end of the cotton swab. 6-0 silk suture material can be seen at the site of implantation. 

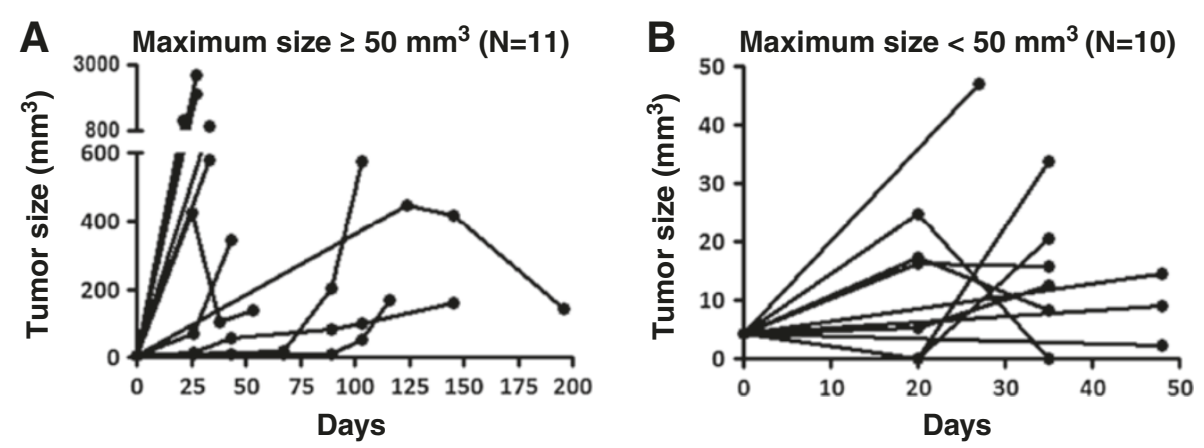

Figure 3 Natural history of xenograft tumor progression based upon serial ultrasound imaging. Based upon maximum tumor size achieved, tumors were sorted into two groups with distinct tumor growth patterns. A. Tumors with a maximum tumor size $\geq 50 \mathrm{~mm}^{3}(\mathrm{~N}=11)$. B. Tumors with a maximum tumor size $<50 \mathrm{~mm}^{3}(\mathrm{~N}=10)$.

tumor sizes of 5 mice that died or were sacrificed within 2 weeks of their last US. There was no statistically significant difference between the groups (US: $524.3 \pm 134.6$ $\mathrm{mm}^{3}$ vs. $457.2 \pm 119.4 \mathrm{~mm}^{3}, \mathrm{P}=\mathrm{NS}$, Figure 4F).

\section{Histological analyses of GIST PDXs}

To investigate if PDXs maintain human GIST tumor properties after implanting tumor into mice (i.e., P0) or after passage once into additional mice (i.e., P1), six mice were sacrificed and their tumor tissues were subject to GIST histopathological analyses and KIT immunohistochemical staining. Five of the six (83.3\%) maintained strong KIT staining of the tumors. It is notable that the hallmarks of tumor necrosis were not seen in the one spindle cell neoplasm lacking KIT expression. Thus, the mechanism for KIT downregulation remains unknown. Despite the presence of tumors, 4 mice were not evaluable histologically due to tissue necrosis overnight.
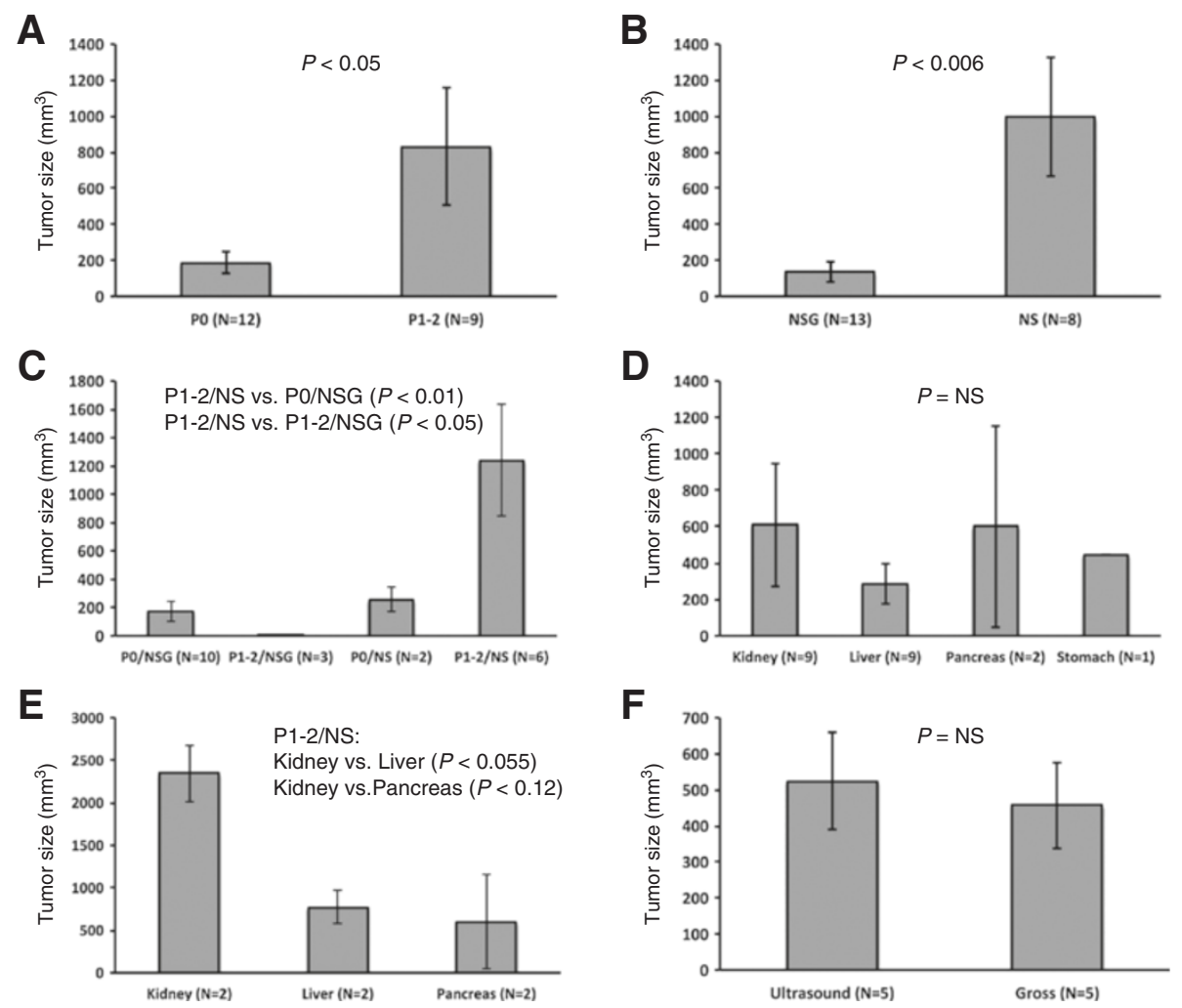

Figure 4 Maximum xenograft size depends upon tumor passage, immunodeficient mouse type, and tumor implantation site.

A. Comparison of tumor passages (PO vs. P1-2). B. Comparison of NSG and NS immunodeficient mice. C. Subgroup comparison of passages plus NSG or NS immunodeficient mice. D. Comparison of tumor implantation sites. E. Comparison of tumor implantation sites in P1-2/NS mice. F. Comparison of gross tumor size at necropsy with ultrasound findings obtained within 2 weeks of death. 
Another 8 mice had tumors, which did not reach our set threshold size of $2500 \mathrm{~mm}^{3}$ for sacrifice and passage, became quite ill due to the Staphylococcal epidemic in our vivarium. We prematurely sacrificed these mice and the tumors tissues were used for passaging to additional healthy mice, leaving no tissue for additional histological analyses. However, this suggests that even in the event of an infection or illness, tumors can be salvaged for additional passaging and study. An example of a P0 mouse with GIST histopathology and KIT staining is shown in Figure 5. At 21.1 weeks, this P0 mouse had an $8.5 \times 7 \times 6.5 \mathrm{~mm}$ tumor in the liver on gross examination (Figure 5A). To verify the primary tumor histologically, serial sections of tumor tissues were stained by $H \& E$ and blindly reviewed by a pathologist (MP). It was evident that a spindle cell neoplasm was present in the primary tumor but not in the neighboring liver tissue (Figure 5B). Furthermore, in contrast to the adjacent non-neoplastic liver that lacked KIT staining, the implanted tumor had strong KIT immunostaining signals (Figure 5C).

\section{PET imaging of GIST PDXs}

PET scan was employed to assess xenografts for human GIST tumor properties. Two mice with tumors from the patient 1's FDG-avid tumor (Figure 1) were evaluated with PET scan and both tumors were FDG-avid on PET. As shown in Figure 6, a P0 mouse had tumor implanted onto the right renal capsule and was subject to PET scan at 16.1 weeks. The xenograft measured $12 \times 10.5 \mathrm{~mm}$ on gross examination (Figure 6B) and was FDG-avid $\left(\mathrm{SUV}_{\max } 2.2, \mathrm{SUV}_{\text {min }} 1.8, \mathrm{SUV}_{\text {mean }} 2.0\right)$ by PET scan as indicated by the arrow in Figure 6A. The FDG uptake in the heart and the brown fat of the shoulder girdles serve as a positive control. Taken together, orthotopic GIST PDXs maintain growth capacity and properties similar to that of patients' GIST tumors.

\section{Discussion}

For the first time, we report an orthotopic patientderived xenograft (PDX) model of human GIST. This model was developed in immunodeficient mice, including the NOD-scid (NS) and the NOD-scid gamma (NSG) strains. In our study, we report an $84 \%$ xenograft success rate as a proof of concept with respect to our novel approach to studying GIST. In both strains, we demonstrate that multiple intraperitoneal sites are capable of supporting GIST growth, with the liver and renal capsule allowing for high rates of engraftment. Moreover, we effectively passaged PDXs at high engraftment rates and demonstrated that high-resolution ultrasound imaging can be employed to serially follow the natural history of tumor growth. Furthermore, tumors passed from NSG mice into the renal capsules of NS mice appear to develop the most robust tumors. Finally, xenografted tumors maintain properties comparable to that of patient's GIST tumor tissue, including cellular histology, KIT expression and FDG-avidity on PET scan.

Tumor xenografts are frequently established by subcutaneous (SQ) injection of immortalized cell lines between the dermis and underlying muscle within the flank, back or footpad of immunodeficient mice [21,33]. For over 30 years, this model has been widely used in cancer research because it is fast, cheap, reproducible, and has been considered sufficient for assessing the activity of anti-tumor agents. It also does not require expensive imaging modalities such as US, computed tomography (CT) or PET in order to visualize tumors because they can be merely measured with calipers [34]. However, these models often fail to accurately predict responses in humans since the SQ microenvironment is not relevant to the sites of primary or metastatic disease [35]. These observations have suggested that such tumor models do not represent appropriate sites for modeling human malignancies in order to evaluate responses to anti-cancer drugs [36].

Given these deficiencies in SQ models, orthotopic tumor xenografts are increasingly being utilized to develop a model with superior clinical relevance and translation applications because these models provide: 1) a biologically relevant site for tumor-host interactions; 2)
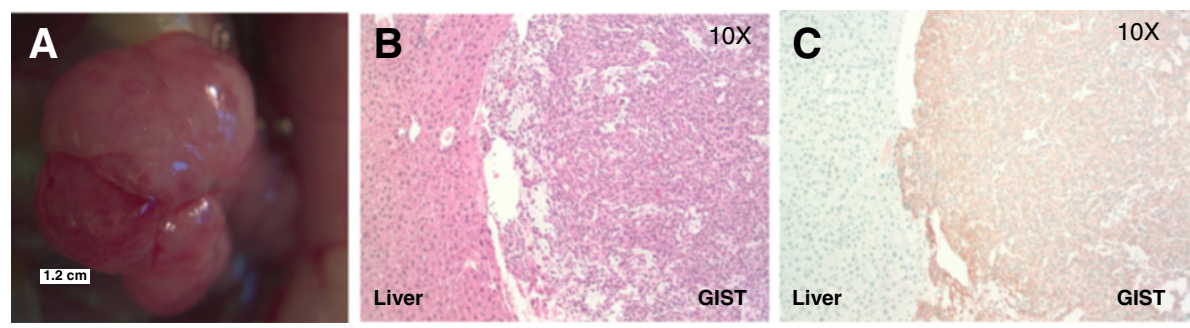

Figure 5 Xenograft tumor progression is marked by tumor growth and KIT immunostaining of a spindle cell neoplasm. A. At 21.1 weeks, this P0 mouse was sacrificed for tumor passage. The primary tumor in the liver measured $8.5 \times 7 \times 6.5 \mathrm{~mm}$ on gross examination. B. Blinded review was performed by a pathologist. H\&E staining confirmed the presence of a spindle cell neoplasm consistent with a GIST. This tumor appears to invade into the liver parenchyma. C. Positive KIT immunostaining was present in the xenografted tumor but not in the adjacent non-neoplastic liver. 


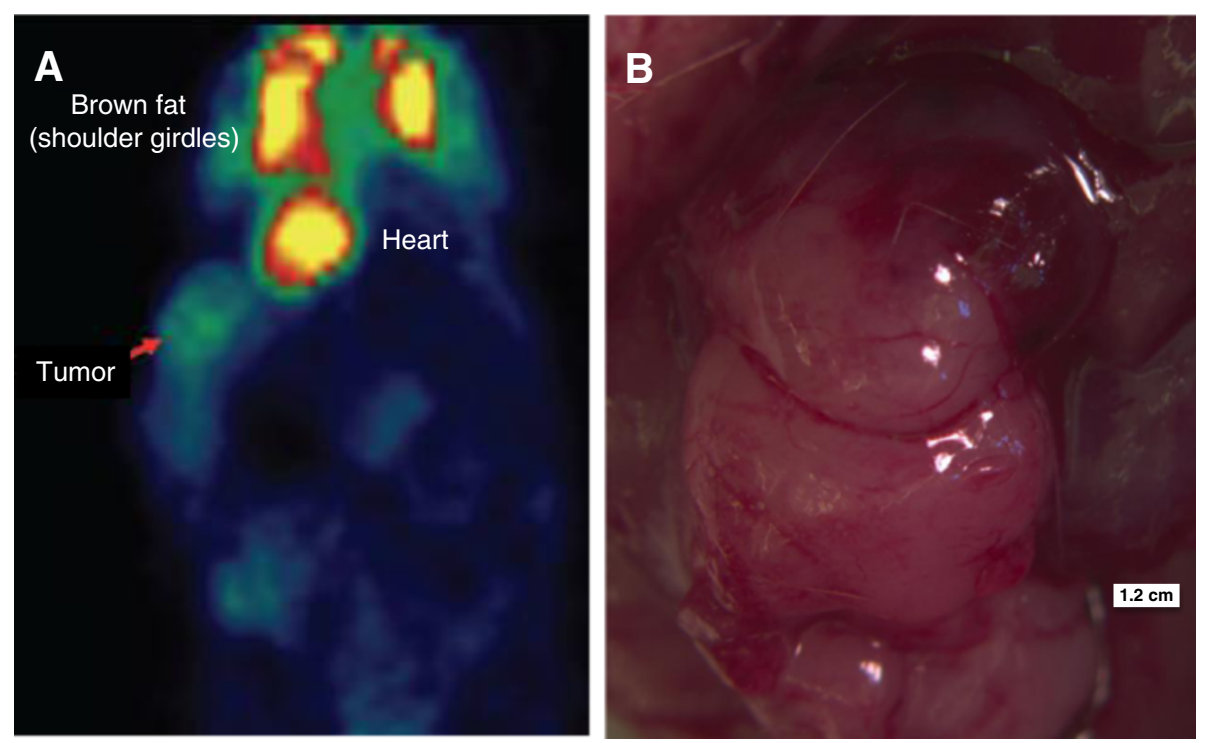

Figure 6 Human GIST xenografts are FDG-avid on PET scan. A. This P0 mouse underwent tumor xenografting into the right renal capsule. At 16.1 weeks after implantation, we performed imaging via PET scan. The arrow denotes tumor uptake of FDG (SUV $V_{\max } 2.2, S U V_{\text {min }} 1.8$, SUV mean 2.0$)$ by PET scan as indicated by the arrow in $\mathbf{A}$. The FDG uptake in the heart and the brown fat of the shoulder girdles serve as a positive control. B. Gross photograph of the corresponding FDG-avid tumor measuring $12 \times 10.5 \mathrm{~mm}$ in the same mouse.

the potential to develop of disease-relevant metastatic progression; 3) the ability to study site-specific dependence upon therapy; and finally, 4) organ-specific expression of genes [36]. While this approach has clear advantages as compared to SQ models, it is undoubtedly more expensive, labor intensive, technically challenging, and requires longer post-procedural healing and recovery $[35,36]$. Nonetheless, orthotopic tumor models have emerged as the preference for many cancer researchers.

To better approximate the genetic heterogeneity of human cancer, PDXs are now emerging as an alternative to cell lines. Like many tumors, GISTs can be SQ implanted into the flanks of mice [21-23,37,38]. However, for the aforementioned reasons, most SQ models are unable to recapitulate human tumor biology and therefore have less clinical relevance [39]. While low passage PDXs have the advantage of maintaining the tumor's complex genetic and epigenetic abnormalities, growing them in a foreign tumor microenvironment (i.e., subcutaneously) partially negates this advantage [35]. In contrast, our xenograft model is a reproducible model of human GIST that replicates the intraperitoneal microenvironment and heterogeneity of human GISTs while allowing for the development of models (i.e., KIT exon 9 mutated GIST) that are not currently available for study in GIST cells or transgenic mouse models. Evidence also suggests that, as opposed to SQ injections, orthotopic xenografts allow for greater invasion into nearby organs, as well as, metastases to the liver [40]. In fact, we observed that GIST PDXs could grow and invade into adjacent tissues, such as the liver (Figure 5B). We have not yet observed metastases, a fact that may have been in part due to rapid local tumor progression that necessitated sacrifice of animals in compliance with IACUC regulations. Nevertheless, our model and other orthotopic xenografts provide a more pathophysiologically relevant environment for tumor growth. We believe this model can be adopted to generate new GIST models (i.e., KIT exon 9 mutants, PDGFR $\alpha$ mutants, and BRAF ${ }^{\mathrm{V} 600 \mathrm{E}}$ mutants), as well as tumors from non-gastric sites such as the small bowel, colon, rectum, esophagus, liver, and peritoneum. However, as previously noted, this model is limited by significant expense and labor utilization, as well as technical procedural challenges, the requirement for expertise in US, and the requirement for longer post-procedural healing and recovery. These factors all contributed to the modest size of our cohort.

In addition to the ability to study tumor biology, such a model can be applied for drug screening. Imatinib is considered the first line of treatment for GIST patients [41]. Unfortunately, once patients develop primary- or secondary-resistance to this drug, there are limited treatment options. One immediate potential application for our orthotopic GIST PDXs is the ability to test agents for efficacy in the setting of imatinib resistance. Hidalgo et al. reported results from their orthotopic model studies with advanced solid tumors obtained from 14 patients that were implanted into immunodeficient mice $[42,43]$. Once tumors were established, the mice were treated with 63 drugs in 232 treatment plans. From this murine "clinic trial," it was determined that there exists a correlation between orthotopic PDX killing and clinical efficacy. All 
drugs maintained their same profile with respect to resistance and sensitivity. The data suggests that individual patient PDXs can be used to personalize a precision treatment approach to treating malignancies. Based upon our findings, a GIST phenotype can be maintained after at least 2 passages in our model. Earlier work by Revehim et al. demonstrated that mutations in KIT exons 11 and 17 were the same in the primary tumor and subcutaneous xenografts after multiple passages in athymic nude mice [44].

\section{Conclusions}

In conclusion, we report the first orthotopic patientderived xenograft model of human GIST. This novel approach provides a reproducible model of human GIST that utilizes the intraperitoneal microenvironment and maintains the genetic heterogeneity of a human gastrointestinal sarcoma. This xenograft model may enhance our ability to study GIST biology in vivo and serve as a preclinical platform for testing novel biomarkers and therapeutics that can inform clinical trial design.

\section{Abbreviations \\ CT: Computed tomography; DMEM: Dulbecco's modification of eagle's medium; DOG-1: Discovered on gastrointestinal stromal tumor 1; FDG: Fluorodeoxyglucose; FFPE: Formalin-fixed, paraffin-embedded; GIST: Gastrointestinal stromal tumor; H\&E: Hematoxylin and eosin; HEPA: High-efficiency particulate air; hpf: High-power fields; \\ IACUC: Institutional animal care and use committee; IP: Intraperitoneal; IRB: Institutional review board; NS: NOD-scid; NSG: NOD-scid gamma; PDGFRa: Platelet-derived growth factor receptor a; PDX: Patient-derived xenograft; PET: Positron emission tomography; SQ: Subcutaneous; SUV: Standardized uptake value; UCSD: University of California, San Diego; US: Ultrasonography; VEGFR: Vascular endothelial growth factor receptor.}

\section{Competing interests}

Jason Sicklick received honorarium from Novartis Pharmaceuticals Corporation for advisory board consultancy and speaking, as well as reimbursement for travel, lodging, and meals. No additional authors have conflicts of interest to declare.

\section{Authors' contributions}

Conception and design: JKS, RS, AML. Acquisition of data: JKS, SYL, MLB, CMT, ESM, RPF, DVJ, MP. Analysis of data: JKS, SYL, CMT, CKH, MP. Interpretation of data: JKS, SYL, CMT, CKH, MP, AML. Drafting of manuscript: JKS, SYL, MLB, CMT. Critical revision of manuscript: JKS, SYL, MLB, CMT, AML. Final approval of the version to be published: JKS, SYL, MLB, CMT, ESM, RPF, DVJ, CKH, MP, RS, AML.

\section{Acknowledgements}

We thank Jim Salinas, Edith Garcia-Gonzalez, and Peter Chase for assistance with tumor procurement, as well as the staff of the In Vivo Imaging Shared Resource of the UCSD In Vivo Cancer and Molecular Imaging Center (P50 CA128346) including Dr. David R. Vera, Jacqueline A. Corbeil, Salman Farshchi-Heydari, Christopher Barback, and Zhengtao Qin.

\section{Grant support}

This work was supported by the Jack Kiel Wolf Memorial Research Fund (JKS) and the Stuart Manroel Memorial Research Fund (JKS), as well as funding provided by UCSD Moores Cancer Center.

\section{Author details}

${ }^{1}$ Division of Surgical Oncology and Department of Surgery, Moores UCSD Cancer Center, University of California, San Diego, 3855 Health Sciences Drive, Mail Code 0987, La Jolla, CA 92093-0987, USA. ²Division of Nuclear Medicine, Moores Cancer Center, and the UCSD In Vivo Cancer \& Molecular Imaging Program, University of California, San Diego, La Jolla, CA, USA.
${ }^{3}$ Department of Pathology, University of California, San Diego, La Jolla, CA, USA. ${ }^{4}$ Division of Medical Oncology and Department of Internal Medicine, Moores UCSD Cancer Center, University of California, San Diego, La Jolla, CA, USA.

Received: 4 November 2013 Accepted: 6 February 2014 Published: 10 February 2014

\section{References}

1. Reichardt $P$, et al: Gastrointestinal stromal tumors I: pathology, pathobiology, primary therapy, and surgical issues. Semin Oncol 2009, 36:290-301.

2. Liegl-Atzwanger B, Fletcher JA, Fletcher CD: Gastrointestinal stromal tumors. Virchows Arch 2010, 456:111-127.

3. Agaram NP, et al: Novel V600E BRAF mutations in Imatinib-naive and Imatinib-resistant gastrointestinal stromal tumors. Genes Chrom Cancer 2008, 47:853-859.

4. Gastrointestinal Stromal Tumor Meta-Analysis Group (MetaGIST): Comparison of two doses of Imatinib for the treatment of unresectable or metastatic gastrointestinal stromal tumors: a meta-analysis of 1,640 patients. J Clin Oncol 2010, 28:1247-1253.

5. Buchdunger E, O'Reilly T, Wood J: Pharmacology of Imatinib (STI571). Eur J Cancer 2002, 38(Suppl 5):S28-S36.

6. Kingham TP, DeMatteo RP: Multidisciplinary treatment of gastrointestinal stromal tumors. Surg Clin North Am 2009, 89:217-233.

7. Dematteo RP, et al: Adjuvant Imatinib mesylate after resection of localised, primary gastrointestinal stromal tumour: a randomised, double-blind, placebo-controlled trial. Lancet 2009, 373:1097-1104

8. Debiec-Rychter M, et al: Use of c-KIT/PDGFRA mutational analysis to predict the clinical response to Imatinib in patients with advanced gastrointestinal stromal tumours entered on phase I and II studies of the EORTC Soft Tissue and Bone Sarcoma Group. Eur J Cancer 2004, 40:689-695.

9. Heinrich $\mathrm{MC}$, et al: Molecular correlates of Imatinib resistance in gastrointestinal stromal tumors. J Clin Oncol 2006, 24:4764-4774.

10. Agaram NP, et al: Pathologic and molecular heterogeneity in Imatinib-stable or Imatinib-responsive gastrointestinal stromal tumors. Clin Cancer Res 2007, 13:170-181.

11. Prenen $\mathrm{H}$, et al: Efficacy of the kinase inhibitor SU11248 against gastrointestinal stromal tumor mutants refractory to Imatinib mesylate. Clin Cancer Res 2006, 12:2622-2627.

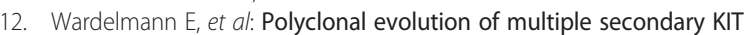
mutations in gastrointestinal stromal tumors under treatment with Imatinib mesylate. Clin Cancer Res 2006, 12:1743-1749.

13. Demetri GD, et al: Efficacy and safety of Sunitinib in patients with advanced gastrointestinal stromal tumour after failure of Imatinib: a randomised controlled trial. Lancet 2006, 368:1329-1338.

14. George S, et al: Clinical evaluation of continuous daily dosing of Sunitinib malate in patients with advanced gastrointestinal stromal tumour after Imatinib failure. Eur J Cancer 2009, 45:1959-1968.

15. Trent JC, et al: A Phase II study of dasatinib for patients with Imatinib-resistant gastrointestinal stromal tumor (GIST). J Clin Oncol 2011, l:Abstract \#10006.

16. George S, et al: A multicenter phase II study of regorafenib in patients (pts) with advanced gastrointestinal stromal tumor (GIST), after therapy with Imatinib (IM) and sunitinib (SU). J Clin Oncol 2011, I:Abstract \#10007.

17. Demetri GD, et al: Randomized phase III trial of regorafenib in patients (pts) with metastatic and/or unresectable gastrointestinal stromal tumor (GIST) progressing despite prior treatment with at least Imatinib (IM) and sunitinib (SU): GRID trial. J Clin Oncol 2012, 30:Abstract \#LBA10008.

18. Corless $\mathrm{CL}$, Barnett $\mathrm{CM}$, Heinrich MC: Gastrointestinal stromal tumours: origin and molecular oncology. Nat Rev Cancer 2011, 11:865-878.

19. Taguchi T, et al: Conventional and molecular cytogenetic characterization of a new human cell line, GIST-T1, established from gastrointestinal stromal tumor. Lab Invest J Tech Methods Pathol 2002, 82:663-665.

20. Tuveson DA, et al: STI571 inactivation of the gastrointestinal stromal tumor c-KIT oncoprotein: biological and clinical implications. Oncogene 2001, 20:5054-5058.

21. Prenen $\mathrm{H}$, et al: Establishment of a mouse gastrointestinal stromal tumour model and evaluation of response to Imatinib by small animal positron emission tomography. Anticancer Res 2006, 26:1247-1252.

22. Huynh $\mathrm{H}$, et al: Sorafenib induces growth suppression in mouse models of gastrointestinal stromal tumor. Mol Cancer Therap 2009, 8:152-159. 
23. Wang TB, Huang WS, Lin WH, Shi HP, Dong WG: Inhibition of KIT RNAi mediated with adenovirus in gastrointestinal stromal tumor xenograft. World J Gastroenterol: WJG 2010, 16:5122-5129.

24. Rubin BP, et al: A knock-in mouse model of gastrointestinal stromal tumor harboring kit K641E. Cancer Res 2005, 65:6631-6639.

25. Sommer $\mathrm{G}$, et al: Gastrointestinal stromal tumors in a mouse model by targeted mutation of the Kit receptor tyrosine kinase. Proc Natl Acad SCi USA 2003, 100:6706-6711.

26. Shultz LD, Ishikawa F, Greiner DL: Humanized mice in translational biomedical research. Nat Rev Immunol 2007, 7:118-130.

27. Ishikawa F, et al: Development of functional human blood and immune systems in NOD/SCID/IL2 receptor gamma chain(null) mice. Blood 2005, 106:1565-1573.

28. Shultz LD, et al: Human lymphoid and myeloid cell development in $\mathrm{NOD} / \mathrm{LtSz}$-scid IL2R gamma null mice engrafted with mobilized human hemopoietic stem cells. J Immunol 2005, 174:6477-6489.

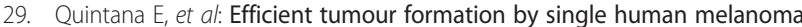
cells. Nature 2008, 456:593-598.

30. King $M$, et al: A new Hu-PBL model for the study of human islet alloreactivity based on NOD-scid mice bearing a targeted mutation in the IL-2 receptor gamma chain gene. Clin Immunol 2008, 126:303-314.

31. Simpson-Abelson MR, et al: Long-term engraftment and expansion of tumor-derived memory $T$ cells following the implantation of non-disrupted pieces of human lung tumor into NOD-scid IL2Rgamma (null) mice. J Immunol 2008, 180:7009-7018.

32. Feldman J, Goldwasser R, Mark S, Schwartz J, Orion I: A mathematical model for tumor volume evaluation using two-dimensions. J Appl Quant Methods 2009, 4:455-461.

33. Edris B, et al: Anti-KIT monoclonal antibody inhibits Imatinib-resistant gastrointestinal stromal tumor growth. Proc Natl Acad Sci USA 2013, 110:3501-3506.

34. Reynolds CP, Sun BC, DeClerck YA, Moats RA: Assessing growth and response to therapy in murine tumor models. Methods Mol Med 2005, 111:335-350.

35. Huynh AS, et al: Development of an orthotopic human pancreatic cancer xenograft model using ultrasound guided injection of cells. PloS one 2011, 6:e20330.

36. Killion JJ, Radinsky R, Fidler IJ: Orthotopic models are necessary to predict therapy of transplantable tumors in mice. Cancer Metast Rev 1998, 17:279-284.

37. Revheim ME, et al: Intermittent and continuous Imatinib in a human GIST xenograft model carrying KIT exon 17 resistance mutation $\mathrm{D} 816 \mathrm{H}$. Acta Oncol 2013, 52:776-782

38. Moriyama $\mathrm{M}$, et al: Establishment and characterization of a novel xenograft model of human gastrointestinal stromal tumor in mice. Anticancer Res 2013, 33:175-181.

39. Richmond A, SU Y: Mouse xenograft models vs GEM models for human cancer therapeutics. Dis Models Mechan 2008, 1:78-82

40. Morioka CY, Saito S, Ohzawa K, Watanabe A: Homologous orthotopic implantation models of pancreatic ductal cancer in Syrian golden hamsters: which is better for metastasis research-cell implantation or tissue implantation? Pancreas 2000, 20:152-157.

41. Sicklick JK, Lopez NE: Optimizing surgical and Imatinib therapy for the treatment of gastrointestinal stromal tumors. J Gastroint Surg 2013, 17(11):1997-2006.

42. Hidalgo $\mathrm{M}$, et al: A pilot clinical study of treatment guided by personalized tumorgrafts in patients with advanced cancer. Mol Cancer Therap 2011, 10:1311-1316.

43. Moro $M$, et al: Patient-derived xenografts of non small cell lung cancer: resurgence of an old model for investigation of modern concepts of tailored therapy and cancer stem cells. J Biomed Biotechnol 2012 2012:568567.

44. Revheim ME, et al: Establishment and characterization of a human gastrointestinal stromal tumour (GIST) xenograft in athymic nude mice. Anticancer Res 2009, 29:4331-4336.

doi:10.1186/1479-5876-12-41

Cite this article as: Sicklick et al:: Generation of orthotopic patient-derived xenografts from gastrointestinal stromal tumor. Journal of Translational Medicine 2014 12:41

\section{Submit your next manuscript to BioMed Central and take full advantage of:}

- Convenient online submission

- Thorough peer review

- No space constraints or color figure charges

- Immediate publication on acceptance

- Inclusion in PubMed, CAS, Scopus and Google Scholar

- Research which is freely available for redistribution 